\author{
Scientific Electronic Archives \\ Issue ID: Sci. Elec. Arch. Vol. 14 (12) \\ December 2021 \\ DOI: http://dx.doi.org/10.36560/141220211448 \\ Article link: https://sea.ufr.edu.br/SEA/article/view/1448
}

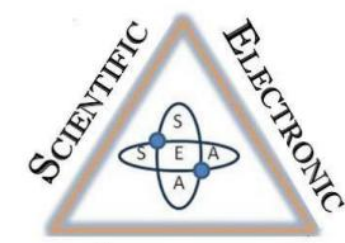

ArChives

ISSN 2316-9281

\title{
Tomografia no estadiamento de neoplasia mamária em cão macho com invasão neoplásica em veia cava caudal - relato de caso
}

\section{Tomography in the staging of breast cancer in a male dog with neoplastic invasion in the caudal vena cava: case report}

\author{
Corresponding author \\ Élide Tibaldi Reis \\ Universidade Federal de Mato Grosso - Campus Sinop \\ elidetibaldi@gmail.com \\ Bruno Gomes de Castro \\ Universidade Federal de Mato Grosso - Campus Sinop
}

\begin{abstract}
Resumo. Dentre os exames complementares fundamentais no diagnóstico de neoplasias, a tomografia computadorizada se destaca devido sua capacidade de formar imagens de forma tridimensional e de retirar a sobreposição através de cortes finos. Por criar imagens dos tecidos com exatidão torna-se aliada na identificação de trombos neoplásicos em grandes vasos. O presente trabalho teve por objetivo analisar o uso da tomografia computadorizada no diagnóstico de neoplasia e de seu estadiamento através do relato de caso de um canino acompanhado durante o estágio supervisionado obrigatório, com carcinoma mamário em região prepucial e durante uma pesquisa de metástase visualizou invasão em veia cava caudal. Concluiu-se que é satisfatório a utilização da tomografia na detecção de invasões neoplásicas, além de agregar no monitoramento e estadiamento de neoplasias. É fundamental reforçar aimportância do diagnóstico histopatológico em casos de tumores, que identificou carcinoma em tumor misto em macho canino, fato considerado raro.
\end{abstract}

Palavras-chaves Tomografia, invasão neoplásica, veia cava.

Abstract. Among the fundamental complementary exams in the diagnosis of neoplasms, tomography computer technology stands out due to its ability to form images in a three-dimensional way and to remove the overlap through fine cuts. For accurately imaging fabrics it becomes an ally in the identification of neoplastic thrombi in large vessels. The present work aimed to analyze the use of computed tomography in the diagnosis of neoplasia and its staging through the case report of a canine followed during the mandatory supervised internship, with breast carcinoma in the preputial region and during a metastasis research visualized invasion in the caudal vena cava. It was concluded that the use of tomography in the detection of neoplastic invasions is satisfactory in addition to adding to the monitoring and staging of neoplasms. It is essential to reinforce the importance of histopathological diagnosis in cases of tumors, which identified carcinoma in mixed tumor in a male canine, which is considered rare.

Keywords: Tomography, neoplastic invasion, vena cava.

\section{Introdução}

As diferentes ferramentas do diagnóstico por imagem adquiriram atuação relevante na oncologia através do diagnóstico e estadiamento de neoplasias (LORIGADOS et al., 2013). A Tomografia Computadorizada (TC) amplia as possibilidades diagnósticas por presentar as estruturas de forma tridimensional, retirando a sobreposição através de cortes finos, o que facilita a análise se comparada com a radiografia convencional. Esses benefícios ocorrem devido a resolução de contraste, através dela é possível criar a imagem de determinado tecido com exatidão devido sua atenuação de raios $X$ conforme suas características físicas e bioquímicas (THRALL, 2014).

Os exames complementares são fundamentais para o diagnóstico de neoplasias, entre eles, sobressai a tomografia computadorizada, a radiografia e a ultrassonografia abdominal. No entanto, o diagnóstico definitivo deve ser feito por análise histopatológica (LABELLE et al., 2004; PAZZINI et al. 2015; PEREIRA et al. 2018). 
Em estudo de Garcia et al. (2012), o exame ultrassonográfico foi capaz de determinar malignidade em $100 \%$ dos casos de massas abdominais analisadas, obtendo ainda sensibilidade de $90,48 \%$ e acurácia de $91,67 \%$, evidenciando que é exame de alta aplicabilidade. No entanto, os mesmos autores afirmam que pode ser um grande desafio descobrir a origem e extensão/invasão de grandes massas abdominais, sendo necessário o uso da tomografia computadorizada ou da ressonância magnética. Mayhew et al. (2017) considera que 0 ultrassom demonstra menor sensibilidade na detecção de trombos tumorais quando comparado com TC.

$\mathrm{Na}$ medicina veterinária, o exame radiográfico é o método de maior acesso e utilização na verificação de neoplasias, mas não consegue apurar a extensão tumoral e o envolvimento de tecidos adjacentes da neoplasia. Ainda, em estágios iniciais, o exame radiográfico pode ser confundido com processo infeccioso, esses obstáculos não estão presentes na TC, já que tem alta visibilidade da localização, extensão e severidade da enfermidade, auxiliando no planejamento cirúrgico caso seja necessário (PAVELSKI et al., 2016; PEREIRA et al., 2018; DALECK et al., 2016).

De acordo com Sprenger et al. (2015) e Pereira et al. (2018) dentre as doenças mais comuns em caninos e felinos, destacam-se as neoplasias. Priebe et al. (2011) e Pereira et al. (2018) acreditam que esse fator acontece devido aos avanços no diagnóstico e maior longevidade destes animais que está associada a dieta mais balanceada, vacinação e protocolos terapêuticos mais eficazes.

As neoplasias são classificadas como benignas e malignas. As benignas possuem crescimento lento, contornos regulares, dificilmente resultam em mortalidade e não invadem tecidos vizinhos. Já as malignas são metastáticas, com crescimento acelerado, podendo levar à destruição tecidual e óbito (DIAS et al. 2015; PAZZINI et al. 2015; PEREIRA et al. 2018). No caso de tumores mamários, os malignos têm uma maior frequência (CASSALI et al., 2017).

O carcinoma em tumor misto (CTM) é a neoplasia mamária mais comum, representando aproximadamente $52 \%$ do total das neoplasias em fêmeas (MENDES, 2007). Já em machos é considerado raro, com incidências inferiores a $1 \%$ (REZENDE E GAMA, 2018). Cassali (2017) afirma que uma das causas prováveis de neoplasia mamária em cães é o aumento da expectativa de vida e irregularidades nos hormônios sexuais, porém em machos são necessários mais estudos.

Por histologicamente o CTM ser semelhante a vasos linfáticos e sanguíneos, ele se torna altamente metastático, dentre os órgãos que podem ser acometidos têm-se as glândulas adrenais (FERREIRA, 2017). Estas topograficamente estão localizadas craniomedialmente em relação aos rins, numa posição retroperitoneal no dorso do abdômen, é envolta por uma cápsula e é dividida em cortical e medular (KÖNIG \& LIEBICH, 2011). Segundo Mayhew et al. (2017) em casos de neoplasias em glândula adrenal podem ocorrer formações de trombos.

Sendo assim, objetivou-se analisar o uso da tomografia computadorizada no diagnóstico de neoplasia e de seu estadiamento através do relato de caso de um canino macho clinicamente diagnosticado com carcinoma mamário em região prepucial e suspeita de metástase pulmonar, que durante a tomografia visualizou invasão em veia cava caudal.

\section{Materiais e Métodos}

No dia 04 de dezembro de 2020, realizou-se tomografia de um canino, macho, 10 anos, SRD, castrado e com peso de $28,4 \mathrm{~kg}$. Segundo a tutora, há dois anos percebeu uma massa próxima ao pênis, no entanto, levou seu canino para consulta oncológica apenas no início de 2020, mais de um ano depois. Além de sinais clínicos como polidipsia, cansaço fácil ao se exercitar e redução no apetite.

$\mathrm{Na}$ consulta, realizaram biópsia excisional, obtendo o diagnóstico de carcinoma em tumor misto de mama com caráter delimitado. $O$ veterinário responsável sugeriu remoção cirúrgica e durante os exames pré operatórios, foi encontrado uma estrutura nodular de opacidade líquida/tecidos moles em região de lobo cranial pulmonar de hemitórax esquerdo, medindo em torno de 7,0 x 5,0 $\mathrm{cm}$, sugestivas de doença pulmonar metastática em radiografia de tórax.

Consequentemente foi solicitada e realizada citologia, porém o diagnóstico foi inconclusivo. Com isso, requisitou nova citologia pulmonar guiada (caso tivesse acesso disponível) e TC de região torácica para investigação de massa.

No exame físico o paciente foi considerado com excesso de peso, foi submetido a anestesia com indução anestésica por Propofol e manutenção inalatória por Isoflurano, no decorrer do exame foi aplicado o contraste iodado não-iônico venoso (Omnipaque), sem reação adversa. Na técnica tomográfica usou cortes transversais de $3,0 \mathrm{~mm}$ e intervalo de mesa de $3,0 \mathrm{~mm}$ de espessura.

A impressão diagnóstica da equipe de laudos foi neoformação oval, de captação heterogênea, com áreas hipocaptantes e hipercaptantes no interior, bem delimitada, localizada em topografia de lobo cranial esquerdo do pulmão, sugere acometer também mediastino cranial, mede cerca $5,88 \mathrm{~cm}$ de altura, $3,82 \mathrm{~cm}$ de largura e 7,85 cm de comprimento (Figura 1), com amplo campo para realização da citologia aspirativa.

A coleta foi realizada pela técnica de punção aspirativa por agulha fina (PAAF) utilizando seringa de $10 \mathrm{ml}$ e cateter intravenoso com agulha jelco $22 \mathrm{G}(0,6 \mathrm{~mm} \times 0,9 \mathrm{~mm} \times 25 \mathrm{~mm})$, para 0 procedimento, foi deixado $2-3 \mathrm{ml}$ de ar dentro da seringa, o local da punção foi higienizado com Clorexidina, a estrutura foi localizada com o aparelho de ultrassom, sendo então introduzido o 
jelco acoplado na seringa e puxado o êmbolo com movimentos em leque várias vezes em direção à estrutura. Lentamente o êmbolo foi solto para não perder o material e a agulha retirada. O êmbolo foi empurrado rapidamente com a extremidade da agulha sobre a lâmina de vidro, depositando o conteúdo aspirado sobre a lâmina e repetindo o processo em mais duas lâminas. Na sequência foi feito um "squash", procedimento em que uma lâmina é deslizada com uma leve pressão sobre outra lâmina, com o intuito de espalhar o material biológico aspirado de forma difusa. As amostras foram identificadas e enviadas ao laboratório.

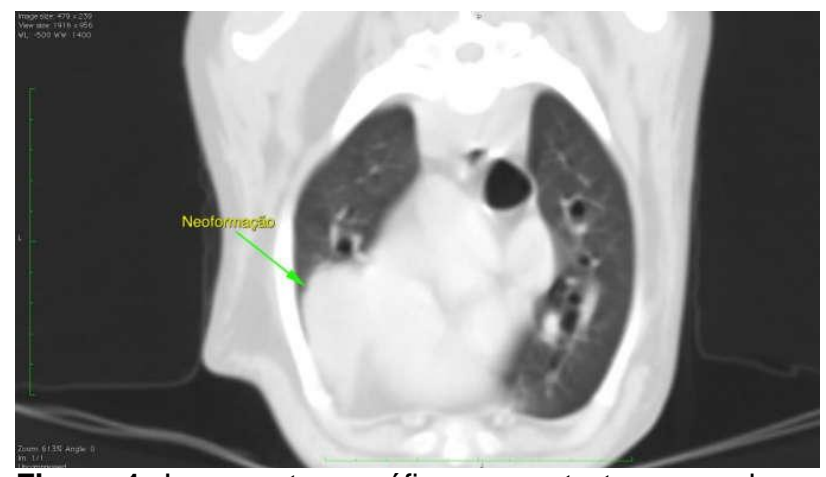

Figura 1. Imagem tomográfica em corte transversal com presença de neoformação medindo cerca de $5,88 \mathrm{~cm}$ de altura $\times 3,82 \mathrm{~cm}$ de largura $\times 7,85 \mathrm{~cm}$ de comprimento em hemitórax esquerdo. Fonte: Advance (Advance, 2021).

$\mathrm{Na}$ citologia foram descritos agregados celulares coesos, com raros neutrófilos e macrófagos no segundo plano. Com caráter de malignidade devido aumento acentuado da relação núcleo citoplasma, basofilia citoplasmática intensa, anisocitose e anisocariose moderadas, modelagem nuclear, presença de nucléolos múltiplos e proeminentes, resultando em carcinoma possivelmente metastático.

Durante a TC de tórax foram avistadas alterações em uma porção hepática. A anestesista responsável pelo exame entrou em contato com o clínico responsável pelo caso sugerindo a tomografia abdominal para melhor elucidação, ele concordou e a tutora autorizou estender o exame para cavidade abdominal.

A mesma técnica tomográfica foi utilizada e foram observadas as seguintes alterações: aumento de volume hepático com contornos abaulados e presença de áreas ovais hipocaptantes ao meio de contraste difusas no parênquima hepático, sendo a maior delas em lobo medial esquerdo medindo cerca de $1,70 \mathrm{~cm}$ de diâmetro; o baço apresentava aumento de volume, com contornos irregulares e presença de nódulo oval hipocaptante contrastado, medindo cerca de $0,47 \mathrm{~cm}$ de diâmetro; presença de neoformação amorfa em topografia de glândula adrenal direita, isodensa, hipocaptante de contraste, com contornos discretamente hipercaptantes, com invasão para o interior da veia cava caudal com obliteração parcial do seu lúmen, medindo cerca de
2,42 cm de altura, 1,34 cm de largura e 4,62 cm de comprimento (Figura 2); por fim, a próstata apresentava contornos irregulares e perda de seu formato anatômico.

Ao final do exame foi indicado exame histopatológico das neoformações abdominais para diagnóstico definitivo.

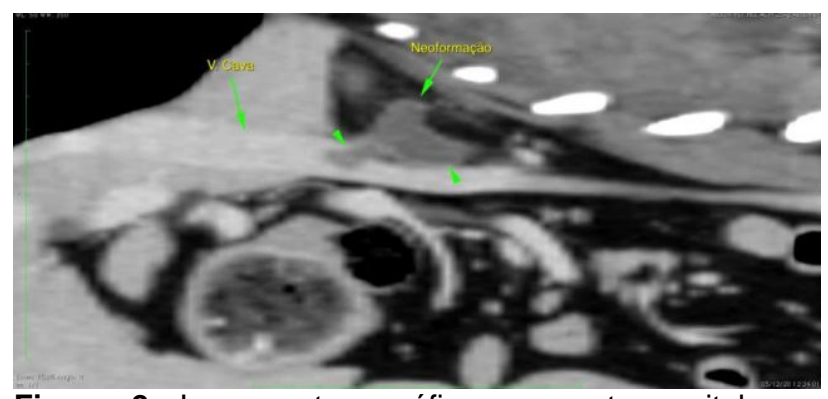

Figura 2. Imagem tomográfica em corte sagital com presença de neoformação com cerca de $2,42 \mathrm{~cm}$ de altura $x 1,34 \mathrm{~cm}$ de largura $x 4,62 \mathrm{~cm}$ de comprimento, invadindo VCC em pontas flechas. Fonte: Advance (Advance,

2021).

\section{Resultados e discussão}

A tomografia computadorizada se mostrou útil na delimitação do tumor, na varredura de metástase, no estadiamento e monitoramento deste estudo, somada a citologia guiada por ultrassom foi possível fechar o diagnóstico do presente relato, o mesmo foi observado no estudo de Lorigados et al. (2013).

O relato de caso abordado trata-se de cão macho acometido por tumor mamário um fator considerado raro pela literatura, visto que de acordo com Oliveira Filho et al. (2010), em estudo com 1.304 cães, somente quatro $(0,3 \%)$ eram machos. Dos 357 cães acometidos por neoplasmas mamários analisados por Mitchell (1974), apenas $1,7 \%$ eram machos. Outros autores afirmam que em comparação com fêmeas a chance de um cão macho ser diagnosticado com tumores mamários é inferior a $1 \%$ em relação às fêmeas (REZENDE \& GAMA, 2018).

Existe alta variação na literatura a respeito da benignidade e da malignidade dos tumores mamários, para Sorenmo et al. (2009) a ocorrência de malignidade é inferior a $54 \%$, já para Martins (2002) essa porcentagem é de 91\%. Em um estudo, de 1465 alterações neoplásicas mamárias em cães, $1.075(73,4 \%)$ eram malignas, com uma relação benigno: maligno de 1: 2,75 (OLIVEIRA FILHO et al. 2010). O canino analisado neste relato tinha CTM que é maligno.

A localização dos tumores mamários em cães machos costuma ser nas mamas abdominais caudais e inguinais, estas, são glândulas apócrinas modificadas, formadas por ductos envoltos por um estroma fibrovascular e adipócitos (REZENDE E GAMA, 2018). Oliveira Filho et al. (2010) acredita que essas mamas têm maior acometimento por terem quantidade de parênquima superior ao das 
outras glândulas mamárias e por possuírem receptores hormonais em abundância, em seu estudo constatou que $61,1 \%$ de 812 casos de tumores mamários estavam acometendo as mamas abdominais caudais e inguinais, o que vem ao encontro do presente estudo.

O CTM por ser histologicamente composto por tecido epitelial e/ou mioepitelial malignos, e mesenquimal benigno, ou misto são altamente metastáticos por estarem ligados a via de vasos linfáticos e sanguíneos, que se direcionam para os linfonodos axilares e inguinais, depois para pulmões, coração, pleura, fígado, diafragma, rins, ossos e glândulas adrenais (FERREIRA, 2017; WITHROW \& VAIL, 2007). De acordo com Moulton et al. (1986) e Oliveira Filho et al. (2010), as incidências em órgãos-alvo de neoplasia mamária são pulmão $85,9 \%$; fígado $23,4 \%$, coração $21,9 \%$, $\operatorname{rim} 20,3 \%$ e adrenal $15,63 \%$. Esses fatores são antagônicos ao que afirma Andrade et al. (2016), sobre a prevalência de metástases em cães machos ser baixa. No presente estudo, estes mesmos órgãos citados acima apresentavam crescimentos anormais, porém somente a massa que estava presente no pulmão foi coletada para análise e as outras neoformações encontradas não teriam área de acesso para citologia sem risco de atingir tecidos possivelmente saudáveis ou grandes vasos.

Ainda em relação a metástase, mais de um tipo histológico de tumor foi identificado em 25,9\% segundo Oliveira et al. (2003), ou seja, isso confirma a necessidade de exames histopatológicos mais detalhados de diferentes regiões.

Bearss et al. (2011) relata que a idade média dos cães machos que desenvolvem neoplasia mamária, quando castrados, é de 9,2 9,4 anos. O paciente estudado era castrado, com idade superior a descrita. No entanto, reafirma a observação de Oliveira Filho et al. (2010) de que a média de idade é entre 10 a 11 anos.

De acordo com a classificação TNM de carcinomas malignos no pulmão da Organização Mundial da Saúde (OMS) que vai de TX a T4, um tumor é considerado T4 quando invade estruturas como mediastino, coração, grandes vasos, traqueia, esôfago, corpo vertebral, carina; se não tiver outras metástases é chamado de $M 0$ e se tiver M1 (EISENBERG, 2004). Sendo assim, por supostamente invadir o mediastino e atingir vários outros órgãos, o tumor metastático visualizado em tomografia torácica seria classificado como T4 M1.

Quanto à neoformação, foi observado no paciente relatado que ela se estendia da glândula adrenal em sentido a veia cava caudal, alterando o lúmen da veia; para Mayhew et al. (2017), esse tipo de alteração em vasos é comum e depende do tipo de tumor.

Diferente do estudo de Silva et al. (2016), em que a maioria das alterações eram em glândulas adrenais esquerdas, nesse estudo o lado direito que foi acometido. O mesmo autor observou que de 100 casos, 43 eram neoplasias e destes apenas dois casos eram carcinoma mamário metastático (SILVA et al., 2016), demonstrando a raridade do caso relatado.

Quando tumores acometem a glândula adrenal, eles se manifestam de modo funcional ou não funcional, comumente os funcionais são metastáticos. Também podem ser classificados como primários, neste caso geralmente são achados de necropsia e representam apenas 1-2\% de todas as neoplasias caninas e $0,2 \%$ das felinas (BERTAZZOLO, et al., 2014; BAILEY \& PAGE, 2007; MELIÁN, 2012). Geralmente nos casos de neoplasias em glândula adrenal, ocorrem formações de trombos, que são mais associados a feocromocitomas, com cerca de $33 \%$ a $55 \%$ dos casos, seguido de tumores adrenocorticais, com aproximadamente $2 \%$ a $22 \%$ dos casos de invasão (incluindo o carcinoma adrenocortical) (MAYHEW et al., 2017). Ainda alguns estudos relatam que a incidência de neoplasias endócrinas em pequenos animais é baixa quando comparada com outros sistemas (SALVADO, 2010; CARVALHO, 2012; MARTINS, 2012). Com isso, se confirmam a necessidade de uma biópsia da área invadida no presente estudo.

Hinrichs et al. (2001) diz que é possível ocorrer hemorragia de adrenal devido aumento da pressão venosa causada pela trombose de veia cava inferior, geralmente ocorre do lado direito. Quando ocorre o carcinoma adrenocortical, problemas clínicos relacionados a secreção de cortisol podem estar presentes, a neoplasia será grande e irregular, com alta metástase em vários órgãos e invasão para veia cava e aorta (LAPERLE \& CAPEN, 2009; SOARES, 2017), sua ocorrência é maior em caninos idosos e bovinos, sendo raro relatos em outras espécies (ECCO \& LANGOHR, 2017). O cão relatado era idoso, apresentava algumas massas grandes, com possíveis metástases e tinha invasão neoplásica em veia cava caudal, encaixa-se nas características citadas.

Os sinais clínicos relacionados a tumores adrenocorticais são poliúria e polifagia em cães, e poliúria, polidipsia associados ou não a diabetes melito secundária em gatos. No exame físico, o abdome estará pênduloso, ganho de peso pode estar associado, assim como fadiga, alterações cutâneas e atrofia muscular (UBUKATA, 2017; DALECK ET AL. 2016; MORRIS \& DOBSON, 2007). Considerando o relato da tutora de polidipsia, cansaço fácil ao se exercitar e redução no apetite, somados ao excesso de peso observado no exame físico, os tumores adrenocorticais entram como possíveis diferenciais no presente relato.

Sousa et al. (2011) diz que tumores com envolvimento venoso costumam ser malignos e que em humanos a taxa de sobrevivência está entre 25 a 18 meses quando o trombo atingia a veia cava inferior, se levar isso em consideração, o paciente tinha chances de sobrevida.

\section{Considerações finais}

$\mathrm{O}$ exame de tomografia computadorizada se mostrou útil na delimitação do tumor, na 
varredura de metástases e importante ferramenta na escolha do possível tratamento. Sua utilização foi satisfatória na detecção de invasões neoplásicas, além de agregar no monitoramento e estadiamento de neoplasias.

É importante reforçar a importância do diagnóstico histopatológico em casos de tumores, que identificou carcinoma em tumor misto em um macho canino, fato considerado raro, sendo assim, é um exame de caráter definitivo para neoplasia.

Não é possível afirmar que os outros tumores encontrados são derivados do CTM por não ter sido realizada a biópsia destes, esse fato aconteceu devido o material estar em locais profundos na cavidade abdominal, com órgãos importantes ao seu redor, podendo causar lesões ou sendo necessário um exame muito incisivo.

\section{Referências}

ADVANCE IMAGEM VETERINÁRIA AVANÇADA, Rio de Janeiro, 2021.

ANDRADE, A. C.; MACHADO, J. R. R.; GRACIANO, K. C.; LUEBKE, G.; KLUTHCOVSKY, L. C.; FILHO, M. M. M. Carcinoma Mamário em Cão Macho - Relato De Caso. Revista Eletrônica Biociências, Biotecnologia e Saúde, Curitiba, n.15, 2016.p.125-157.

BAILEY, D.B; PAGE, R.L. Tumors of endocrine system. In: WITHROW, S.J; VAIL, D.M. Withrow and Mac Ewen's small animal clinical oncology. Elsevier, Missouri, ed.4, 2007. p. 583-603.

BEARSS, J. J.; SCHULMAN, F. Y.; CARTER, D. Características histológicas, imunohistoquímicas e clínicas de 27 tumores mamários em 18 cães machos. Veterinary Pathology, Belo Horizonte, v. 49, n.4, 2011. p. 602-607.

BERTAZZOLO, W.; DELL'ORCO, M.; BONFANTI, U.; GHISLENI, G.; CANIATTI, M.; MASSERDOTTI, C.; ROCCABIANCA, P. Accuracy of cytology in distinguishing adrenocortical tumors from pheochromocytoma in companion animals. Veterinary Clinical Pathology, v. 43, n.3, 2014. p. 453-459.

BORTOLINI, Z.; MATAYOSHI, P. M.; SANTOS, R. V.; DOICHE, D. P.; MACHADO, V. M. V.; TEIXEIRA, C. R.; VULCANO, L. C. Casuística dos exames de diagnóstico por imagem na medicina de animais selvagens - 2009 a 2010. Arq. Bras. Med. Vet. Zootec., Botucatu, v. 65, n.4, 2013. p. 1247-1252.

CARVALHO, G. L. C. Frequência de neoplasias endócrinas em cães e perfil da população afetada em uma rotina de atendimento em endocrinologia. In: TCC (GRADUAÇÃO) - CURSO DE MEDICINA VETERINÁRIA, UNIVERSIDADE FEDERAL DO RIO GRANDE DO SUL, 2012. Porto Alegre: UFRGS, 2012. p. 24.
CASSALI, G. D. Patologia Mamária Canina. 1. ed. São Paulo: Medvet, 2017.

DALECK C.R.; FONSECA C.S.; CANOLA J.C. Oncologia em cães e gatos. Rio de Janeiro: Roca, 2016.

DIAS F.; DIAS L.; PEREIRA L.; CABRINI T.; ROCHA J. Neoplasias orais nos animais de companhia - Revisão de literatura. Revista científica eletrônica de medicina veterinária, v. 20, 2015. p. 19.

ECCO, R.; LANGOHR, I. M. Patologia Veterinária. ed. 2. Rio de Janeiro: Roca, 2017.

EISENBERG, A. L. A. TNM: classificação de tumores malignos. Ministério da Saúde - Secretaria de Atenção à Saúde - Instituto Nacional de Câncer INCA, Rio de Janeiro, 6. ed., 2004. p. 254.

FERREIRA, Q. Q. C. Carcinoma mamário em tumor misto metastático em cão. In: MONOGRAFIA APRESENTADA AO DEPARTAMENTO DE CIÊNCIAS VETERINÁRIAS, DA UNIVERSIDADE FEDERAL DA PARAÍBA. Areia: UFPB, 2017. p. 32.

KÖNIG, H. E.; LIEBICH, H. Anatomia dos Animais Domésticos: Texto e Atlas Colorido. ed. 4, Porto Alegre: Artmed, 2011. p. 586.

GARCIA, D. A. A.; FROES, T. R.; GUÉRIOS, S. D. Ultrassonografia abdominal pré-operatória em cães e gatos com suspeita de tumores abdominais. Ciência Rural, Santa Maria, v.42, n.1, 2012. p. 105111.

LAPERLE, K. M. D.; CAPEN, C.C. Sistema Endócrino. In: MCGAVIN, M. D.; ZACHARY, J.F. BASES DA PATOLOGIA EM VETERINÁRIA. ed. 4, Rio de Janeiro: Elsevier, 2009. p. 695.

LORIGADOS, C. A. B.; MATERA, J. M.; COPPI, A. A.; MACEDO, T. R.; LADD, F. V. L.; SOUZA, V. A. F.; PINTO, A. C. B. C. F. Tomografia computadorizada de mastocitomas em cães: avaliação pré e pós tratamento quimioterápico. Pesquisa Veterinária Brasileira, São Paulo, v. 33, n.11., 2013. p. 1349-1356.

MARTINS, L. M. C. Neoplasias em Felinos: Um estudo descritivo de 3 anos. In: DISSERTAÇÃO (MESTRADO) - CURSO DE MEDICINA VETERINÁRIA, UNIVERSIDADE LUSÓFONA DE HUMANIDADES E TECNOLOGIAS, 2012. Lisboa: ULHT, 2012. p. 61.

MARTINS, A. M. C. R. P. F.; TAMASO, E.; GUERRA, J. L. Retrospective review and systematic study of mammary tumors in dogs and characteristics of the extracellular matrix. Braz. J. Vet. Res. Anim, São Paulo, v.39, n.1, 2002. p. 3842. 
MARTINS, B. D. C.; TORRES, B. B. J.; RODRIGUEZ, A. A. M.; GAMBA, C. O.; CASSALI, G. D.; LAVALLE, G. E.; MELO, E. G. Clinical and pathological aspects of multicentric hemangiosarcoma in a Pinscher dog. Arquivo Brasileiro de Medicina Veterinária e Zootecnia, v. 65, n. 2, 2013. p. 322-328.

MAYHEW, P. D.; CULP, W. T. N.; BALSA, I. M.; ZWINGENBERGER, A. L. Phrenicoabdominal venotomy for tumor thrombectomy in dogs with adrenal neoplasia and suspected vena caval invasion. Veterinary Surgery The American College of Veterinary Surgeons, 2017. p. 1-9.

MENDES, T. C.; GUIM, N. T.; DIAS, M. F.; RAPOSO, J. B.; FERNANDES, C. G. Comparação entre os sistemas histomorfológicos e de graduação histológica para classificação prognóstica de tumores mamários em cadelas. Acta Scientiae Veterinariae, Brasil, v. 35, n. 3, 2007. p. 339-343.

MELIÁN, C. Investigation of Adrenal Masses. In: MOONEY, C. T.; PETERSON, M. BSAVA MANUAL OF CANINE AND FELINE ENDOCRINOLOGY. ed. 4. Ed. Philadelphia: Bsava, 2012. p. 272-277.

MORRIS, J.; DOBSON, J. Oncologia em Pequenos Animais. c. 14. São Paulo: Roca, 2007. p. 217-221.

MOULTON, J. E.; ROSENBLATT; L. S.; GOLDMAN, M. Mammary tumors in a colony of Beagle dogs. Veterinary Pathology, v. 23., n 6., 1986. p. 741-749. 43.

OLIVEIRA FILHO, J. C.; KOMMERS, G. D.; MASUDA, E. K.; MARQUES, B. M. F. P. P.; FIGHERA, R. A.; IRIGOYEN, L. F.; BARROS, C. S. L. Estudo retrospectivo de 1.647 tumores mamários em cães. Pesquisa Veterinária Brasileira, Rio de janeiro, v. 30, n.2, 2010. p. 9.

PAZZINI, J. M.; NARDI, A. B.; SERAFIM, E. L.; CALAZANS, S. G.; HUPPES, R. R.; ROCHA, C. E. B. S.; VIÉRA, R. B.; OLIVA, C. A. C.; BUENO, C.; MARCHIORI, M. G. F. Rinotomia dorsal empregada no tratamento de carcinoma intranasal em dois cães Rhinotomy backbone in treatment in two dogs intranasal carcinoma. Revista Portuguesa de Ciências Veterinárias, Lisboa, v. 110, 2015.

PAVELSKI, M.; SILVA, D. M.; FROES, T. R. Radiografia das cavidades craniana e nasal em afecções neoplásicas em cães: características e limitações. Veterinária e Zootecnia, v. 23, 2016. p. 164-73.

PEREIRA, L. B. S. B.; PESSOA, H. F.; FILHO, L. B. F.; CARVALHO, A. J.; PONTES, M. B.; D'ALCANTARA, N. A. L. G.; LIMA, J. B. O.; ALBUQUERQUE, P. V.; NASCIMENTO, J. C. S.; SOUZA, W. M. A. Neuroblastoma olfatório em cão: achados tomográficos e histopatológicos. PUBVET, Maringá, v.12, n.7, 2018. p. 1-6.

PRIEBE, A.P.S.; RIET-CORREA, G.; PAREDES, L. J. A.; COSTA, M. S. F.; SILVA, C. D. C.; ALMEIDA, M. B. Ocorrência de neoplasias em cães e gatos da mesorregião metropolitana de Belém, PA entre 2005 e 2010. Arquivo Brasileiro de Medicina Veterinária e Zootecnia, Belém, v. 63, 2011. p. 6.

REZENDE, K. K.; GAMA, A. P. A. Diagnóstico de carcinoma mamário em cão macho: relato de caso. Acta Biomedica Brasiliensia, Rio de Janeiro, v. 9, n.2, 2018. p. 4

SALVADO, I. S. S. Estudo retrospectivo das neoplasias em canídeos e felídeos domésticos, analisados pelo laboratório de Anatomia Patológica da Faculdade de Medicina Veterinária da Universidade Técnica de Lisboa, no período compreendido entre 2000 e 2009. Universidade Técnica de Lisboa, Lisboa, 2010. p. 109.

SILVA, E.O.; DI SANTIS, G.W.; HEADLEY, S.A.; BRACARENSE, A.P.F.R.L. Alterações patológicas em adrenais de cães: estudo retrospectivo de 80 casos. In: I CONGRESSO DE PESQUISA EM SAÚDE ANIMAL E HUMANA, LONDRINA, 2016. Presidente Prudente: UNIP, 2016. p. 3.

SOARES, I. M. F. Carcinoma cortical da adrenal não funcional com invasão da veia cava caudal em cão. In: MONOGRAFIA APRESENTADA AO DEPARTAMENTO DE CIÊNCIAS VETERINÁRIAS, DA UNIVERSIDADE FEDERAL DA PARAÍBA. Areias: UFPB, 2017. p. 26.

SORENMO, K. U.; KRISTIANSEN, V. M.; COFONE, M. A.; SHOFER, F. S.; BREEN, A. M.; LANGELAND, M.; MONGIL, C. M.; GRONDAHL, A. M.; TEIGE, J.; GOLDSCHMIDT, M. H. Canine mammary gland tumors: a histological continuum from benign to malignant, clinical and histopathological evidence. Veterinary and Comparative Oncology, Filadélfia, 2009. p. 162-172. 44

SOUSA, R.; PEDRO, L. M.; PEREIRA, S.; CORREIA, H.; FERNANDES, R; GIMENEZ, J. L.; LOPES, T.; GAMA, A. D. Carcinoma de células renais com invasão da veia cava inferior: a propósito de um caso clínico. Angiologia e Cirurgia Vascular, Lisboa, v. 7, n.3, 2011. p. 149 - 159.

SPRENGER, L. K.; RISOLIA, L. W.; GABARDO, L.; MOLENTO, M. B.; SILVA, A. W. C.; SOUSA, R. S. 2015. Tumores neoplásicos de cães e gatos diagnosticados no laboratório de patologia veterinária da Universidade Federal do Paraná. Archives of Veterinary Science, Paraná, v. 20, n. 10, 2015. p. 6. 
REIS et al. Tomografia no estadiamento de neoplasia mamária em cão macho com invasão neoplásica em veia cava caudal: relato de

THRALL, D. E. Diagnóstico de Radiologia Veterinária. ed.6. Rio de Janeiro: Elsevier, 2014. p. 1540-1550.

UBUKATA, R. Neoplasias da Glândula Adrenal. In: DALECK, C. R.; NARDI, A. B. ONCOLOGIA EM CÃES E GATOS. ed.2. Rio de Janeiro: Roca, 2017. p. $445-451$.

WITHROW S.J, MACEWEN E.G. Small animal clinical oncology. 3 ed. Philadelphia: Saunders, 2001. p.356-72. 